\title{
Reorienting with terrain slope and landmarks
}

\author{
Daniele Nardi • Nora S. Newcombe • Thomas F. Shipley
}

Published online: 10 October 2012

(C) Psychonomic Society, Inc. 2012

\begin{abstract}
Orientation (or reorientation) is the first step in navigation, because establishing a spatial frame of reference is essential for a sense of location and heading direction. Recent research on nonhuman animals has revealed that the vertical component of an environment provides an important source of spatial information, in both terrestrial and aquatic settings. Nonetheless, humans show large individual and sex differences in the ability to use terrain slope for reorientation. To understand why some participants - mainly women - exhibit a difficulty with slope, we tested reorientation in a richer environment than had been used previously, including both a tilted floor and a set of distinct objects that could be used as landmarks. This environment allowed for the use of two different strategies for solving the task, one based on directional cues (slope gradient) and one based on positional cues (landmarks). Overall, rather than using both cues, participants tended to focus on just one. Although men and women did not differ significantly in their encoding of or reliance on the two strategies, men showed greater confidence in solving the reorientation task. These facts suggest that one possible cause of the female difficulty with slope might be a generally lower spatial confidence during reorientation.
\end{abstract}

Keywords Individual and sex differences - Spatial abilities . Reorientation · Slope $\cdot$ Geographical slant $\cdot$ Spatial confidence

D. Nardi $\cdot$ N. S. Newcombe $\cdot$ T. F. Shipley

Department of Psychology, Temple University,

Spatial Intelligence and Learning Center,

Philadelphia, PA 19122, USA

D. Nardi $(\bowtie)$

Sapienza Università di Roma,

Via dei Marsi 78,

00185 Rome, Italy

e-mail: d.nardi@uniroma1.it
Few everyday cognitive abilities exhibit individual differences as marked as those seen in navigation tasks. It is apparent that some people are extremely good at finding their way, while others struggle and experience anxiety during the journey (e.g., Ishikawa \& Montello, 2006; Lawton, 1994; Schinazi, Epstein, Nardi, Newcombe, \& Shipley, 2009). The complexity of the navigation process makes it difficult to understand the source of such variability in performance - after all, successful navigation depends on many component skills (e.g., perception, spatial memory, position updating, and construction of a mental map of the environment). Here we focus on the first step that any mobile animal encounters when navigating: establishing where it is and which direction it is facing. This step, often called orientation, or reorientation when orientation has been lost, is accomplished when a navigator identifies a key element of the environment (e.g., a sign, specific landmark, or familiar sound).

A central question in the reorientation literature is whether any of these spatial cues has a more important role (is more salient) than others. For example, at least in small spaces, the geometric shape of the environment determined by bounding walls (e.g., in a room) seems to be a particularly strong type of reorienting cue - so strong that nonhuman animals (Cheng, 1986) and human children (Hermer \& Spelke, 1994, 1996) tend to focus on this cue and disregard other, potentially more useful cues. However, the strength of geometric cues appears to wane as the space grows larger (Learmonth, Nadel, \& Newcombe, 2002), for a variety of reasons that include the potential for action in the space and the distance of the cues from the participant (Learmonth, Newcombe, Sheridan, \& Jones, 2008). Another type of spatial information that has recently been shown to dominate reorientation and goal searching is the vertical extent of the environment. Both in terrestrial environments (for homing pigeons, see Nardi, Nitsch, \& Bingman, 2010; for rats, Jovalekic et al., 2011) and in volumes of water (for fish, see 
Holbrook \& Burt de Perera, 2011), the vertical component of space seems to provide a very salient source of information and to be treated differently from the horizontal dimensions. In particular, pigeons use a uniform, sloping floor to reorient, even if other cues, such as the shape of the environment, are available (Nardi \& Bingman, 2009) and are better predictors of the goal location (Nardi et al., 2010). The strong reliance on slope gradients is probably related to the increased effort of moving on inclines, and to the fact that navigating on tilted surfaces creates a multimodal sensory experience, as slope is simultaneously perceived by visual, kinesthetic, and vestibular receptors.

Despite the strong reliance on slope exhibited by nonhuman animals, the only human study on terrain slope reorientation carried out in a real environment showed surprising variability in performance, with a large percentage of the participants being unable to notice the incline and use it to locate a hidden goal (Nardi, Newcombe, \& Shipley, 2011). Sex was found to be related to these individual differences, with men showing a performance advantage of $1.4 S D$ s over women - an effect size larger than that reported in most studies of mental rotation, a spatial skill characterized by large sex-related differences (Voyer, Voyer, \& Bryden, 1995). Although comparisons with nonhuman animal results are hampered by the use of different inclinations ( $5^{\circ}$ for humans, $20^{\circ}$ for pigeons), the study of Nardi et al. (2011) shows that humans find it difficult to reorient using a slope gradient of moderate magnitude $\left(5^{\circ}\right.$ is a common inclination used in wheelchair ramps). This finding is important because vertically extended surfaces are part of natural environments: The vertical topography of the land (a mountain, a valley) can be used to identify goal locations in the large scale, and - at least in the case of a uniform slope, like the side of a hill - the gradient establishes a stable, small-scale directional reference frame (e.g., "the house is uphill with respect to the school").

To further our understanding of how people reorient by terrain slope and why it is apparently a difficult task, in the present experiment we provided an enriched testing environment as compared to that of Nardi et al. (2011). In the previous study, participants could use only the slope of the floor to reorient. Normally, however, objects are present in the environment and can be used as landmarks to gain a sense of orientation and for encoding a goal location. Thus, in this work, in addition to the slope of the terrain, we included a set of distinct landmarks. Reorientation by landmarks is well established and, at least for human adults, can be considered a simple task (e.g., Ratliff \& Newcombe, 2008). By providing multiple strategies for reorientation-one slope-based and the other landmark-based - the present experiment could tease apart the general ability to reorient from the ability to use slope per se.
Reorientation by slope and landmark cues is a particularly interesting case, because slope and landmark are two distinct classes of spatial cues. A uniform terrain slope falls in the category of directional cues because the gradient provides the navigator with bearing information (like with a compass, north can be likened to uphill, south to downhill, etc.); local landmarks, instead, constitute positional cues, as they supply the navigator with both direction and distance information (e.g., a nonvisible target location can be estimated by a vector centered on a landmark). This distinction has been proposed by Jacobs and Schenk (2003), who also suggested that the sexes (not only in humans) might differently rely on these types of cues: males more on directional cues and females more on positional cues.

The (scarce) existing research on human reorientation and navigation in sloped environments has shown that the presence of a geographical slant $\left(4^{\circ}\right.$ inclination) improves navigation performance in a landmark-rich virtual town (Restat, Steck, Mochnatzki, \& Mallot, 2004); furthermore, it has also provided evidence that, indeed, men tend to focus more than women on directional cues - including, but not limited to, terrain slope $\left(30^{\circ}\right.$ inclination; Chai \& Jacobs, 2009, 2010). Crucially, however, these studies employed virtual environments, which deprive participants of the full set of sensory information available in the real world for the perception of tilt. In the Restat et al. study, the environment was presented on a large screen and participants used a bicycle simulator that enabled slant perception on the basis of effort (through force feedback), but not on the basis of body posture (the tilt of the bicycle did not vary according to the slope); in Chai and Jacobs's $(2009,2010)$ studies, the environment was experienced through desktop monitors, providing only the visual component of slope information. In the present experiment, we used a real sloped enclosure; walking in this apparatus enabled participants to perceive the full set of visual, kinesthetic, and vestibular stimuli associated with tilt. Therefore, by using a real environment and by including landmarks, we were able to examine reorientation with a more naturalistic experience of a sloped terrain. Our main purpose was to assess how people use these two types of cues (slope and landmarks) and whether, using sex as a proxy, there are related individual differences in their use.

Undergraduate students were taken through a reorientation task in which, after being spun on a swivel chair with the blindfold on, they had to find a target hidden in one of the corners of a tilted, square enclosure. In each corner was a black-and-white card with a distinct pattern that could be used to identify the target location; the differences among these patterns were recognizable but subtle, as ascertained through pilot studies, so that the task was not too easy. Therefore, unlike in our previous experiment (Nardi et al., 2011), participants could use both the slope of the floor 
(e.g., "the target is uphill/left") and a set of landmarks (e.g., "the target is near this card, or opposite that other card") to encode the goal (see Fig. 1). The reorientation task was carried out for two reinforced trials in which the goal was always in the same corner (referred to as "training trials"). After this, two types of unreinforced tests were carried out. In the conflict tests, the set of cards and the slope predicted two different target locations, so that participants' choices would reveal their reliance on the landmark strategy or the slope strategy. In the single-cue tests, only one of the cues was available (slope was available in the real-world task, and landmarks were available in a paper-and-pencil version of the task); therefore, it could be deduced whether participants encoded the cue and could use it to reorient.

Most of the experimental paradigms in the literature of reorientation have used discrete choices among distinct, potential target locations (typically, the corners of an enclosure) as the dependent variable (for a review, see Cheng \& Newcombe, 2005). Here participants, in addition to discrete choices, provided also a measure of confidence for each hiding location. In each trial, they had a total of ten confidence points (CPs) that they could allot among as many locations as they wanted-the more CPs a location was assigned, the greater the confidence for that specific location. In this way, we could estimate the level of confidence displayed during the task, and we had a more graded measure for how slope and landmark cues are taken into account in the reorientation process. In particular, as a secondary goal of this study, we could examine whether, during the conflict tests, people showed signs of using a combination of both cues (e.g., spreading the CPs between the locations associated with slope and landmark) or univocally relied on one (e.g., allotting all CPs to one location).

\section{Method}

Apparatus

The apparatus was the same as the one used in Nardi et al. (2011). The experimental enclosure measured $244 \times 244 \mathrm{~cm}$ and was $203 \mathrm{~cm}$ high (see Fig. 1); it was placed inside a room measuring $290 \times 460 \mathrm{~cm}$ that was $250 \mathrm{~cm}$ high. The floor of the enclosure consisted of a wooden platform ( $244 \times$ $244 \mathrm{~cm}, 12 \mathrm{~cm}$ thick), covered by gray carpet. White sheets on a PVC pipe frame composed the walls and the ceiling of the enclosure. The enclosure was tilted at an inclination of $5^{\circ}$ (the same inclination used in Nardi et al., 2011). On the

\section{Top view of the enclosure}

\section{Side view of the enclosure}

PVC pipe frame covered by sheets

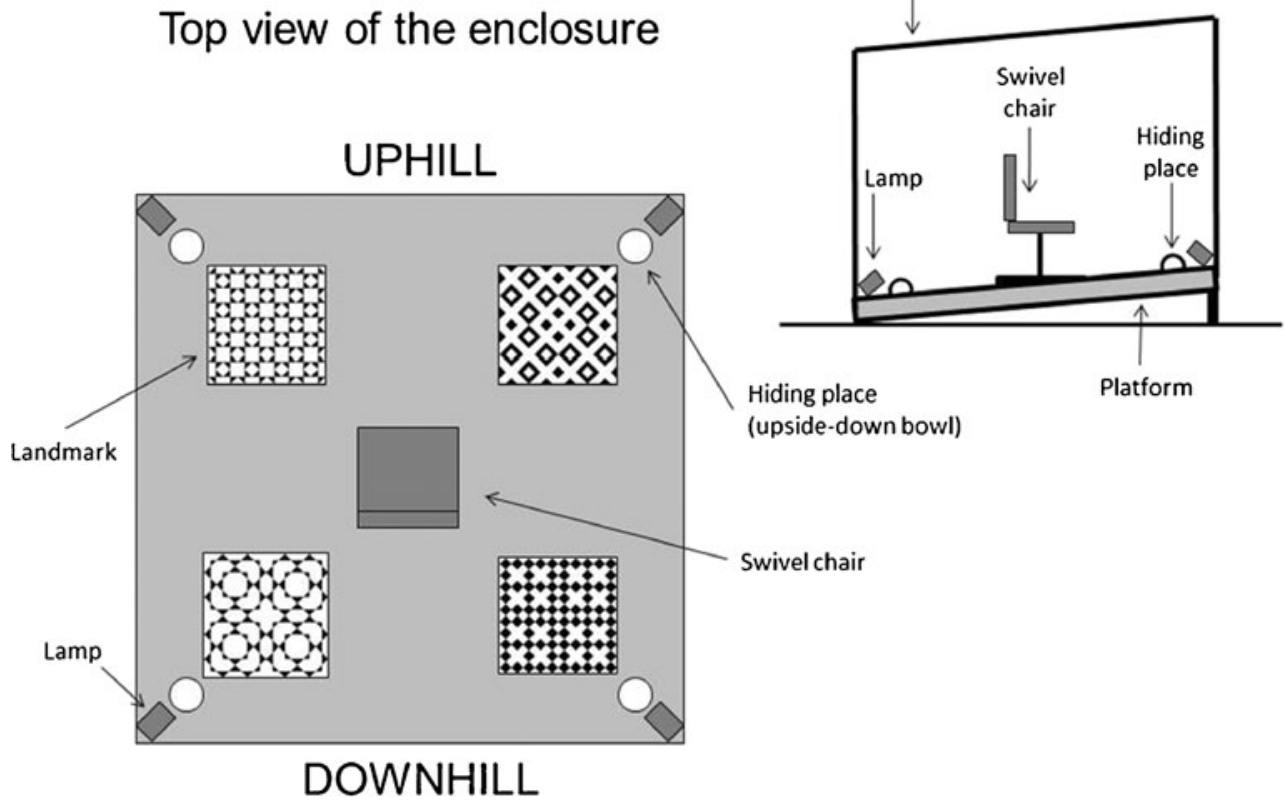

Fig. 1 Schematic representation of the experimental enclosure, viewed from the side and from the top. Four laminated cards with distinct, black-and-white patterns were placed in the corners of the enclosure and could be used as landmarks to determine the target location. The cards are not represented at scale; they are larger here for clarity. The four cards were always arranged in the same configuration, and the one associated with the target was the same for all participants (the card represented here in the upper left corner). During the conflict tests, the cards were rotated clockwise and counterclockwise as a whole array (still preserving their configuration). During the slope test, the cards were removed. During the landmark test, this top view of the enclosure was presented to the participant on a sheet of paper, with this exact configuration of the cards ("uphill" and "downhill" were omitted) 
floor of the enclosure, in each corner was a 25-W lamp (approximate dimensions: $11 \times 11 \mathrm{~cm}, 18 \mathrm{~cm}$ high) and a red bowl placed upside down $(16 \mathrm{~cm}$ in diameter, $8 \mathrm{~cm}$ deep), which constituted the hiding place for the target. In front of the red bowls, on the floor were placed the landmark cues; they were four $11 \times 11 \mathrm{~cm}$ laminated cards with distinct black-and-white patterns (see Fig. 1). A swivel chair was placed in the center of the enclosure (base, $56 \mathrm{~cm}$ in diameter; total height, $110 \mathrm{~cm}$ ). A wedge was placed under the chair such that the chair's axis of rotation was always parallel to the force of gravity. The bottom of the chair was covered with a square piece of white cloth $(61 \times 61 \mathrm{~cm})$ that covered the legs of the chair and the wedge. It is important to note that, when spinning on the swivel chair, the participants' feet never touched the floor, so no cues were available for keeping track of their position relative to the slope.

\section{Participants}

The participants were 40 male and 40 female Temple University undergraduate students, from the ages of 18 to 30 , who volunteered as a means of fulfilling course requirements. The sample included $61.3 \%$ White participants, $16.3 \%$ African-Americans, $12.5 \%$ Asians, $5.0 \%$ Hispanics, $2.5 \%$ of other minorities, and $2.5 \%$ undeclared. The average ages were 21.4 for males $(S D=5.4)$ and 20.5 for females $(S D=2.8)$. Participants signed up for the experiment through a website, on which they were told to wear comfortable shoes and that heels were not allowed. Before starting the experiment, participants signed the consent form.

\section{Procedure}

Training trials Each participant wore a blindfold and was led by the experimenter into the room and into the enclosure. The participant sat on the swivel chair and was spun around for about $30 \mathrm{~s}$. After this, the participant took off the blindfold and was asked to walk around the enclosure to get acquainted with it; this procedure was carried out to facilitate the perception of the slope for the participant. The experimenter then showed the target (a \$1 bill) and said, "I will hide this under one of the bowls in the corners; your job is to try to remember where it is, and after being spun on the chair, you will have to find it." The participant was also told that, apart from making a discrete choice, he or she would have to allot ten confidence points (CPs) on the basis of his or her confidence in each corner. The experimenter said, "if you are completely sure that the target is in one corner, you can give ten points to that corner; if instead you are somehow uncertain between two or more corners, you can subdivide those points, giving more points to the corner you are more confident of." The experimenter then gave a couple of examples and ascertained that the participant understood how to allot the CPs. No instruction was given as to which cue to use to encode the target location, but the experimenter said that, during the disorientation procedure, the curtains and cloth under the chair would be moved; in this way, participants were discouraged from using uncontrolled details (wrinkles, creases) on the sheets or on the cloth under the chair. To encode the goal location, the participants could take as much time and walk as much as they wanted. The experimenter caught the attention of the participant and placed the target under one bowl; when ready, the participant sat on the chair, put on the blindfold, and was disoriented. Spinning occurred for about $1 \mathrm{~min}$, changing direction and speed of rotation. During the disorientation procedure, the experimenter moved the sheets that composed the walls of the enclosure (each sheet was moved to the adjacent side, clockwise) and readjusted the cloth under the chair, in case it had been moved by the participant's feet. After being spun, the participant took off the blindfold, stood up and was asked, "can you tell me where I hid the target"? The participant was told to take as much time and to walk around as much as he or she wanted; when ready, the participant would have to allot the CPs and make the discrete choice, and then would have to walk to the chosen bowl and uncover it to see if the target was there. If the choice was incorrect, the experimenter uncovered the correct bowl and showed the target. This procedure was repeated for another trial (a total of two training trials), with the target always in the same corner (reference memory paradigm). In each trial, the participant started facing a different side of the enclosure (in counterbalanced orders across each sex), and the experimenter always stood at the back of the chair. The location of the target with respect to the slope gradient was counterbalanced across all four corners for each sex. The four cards were always arranged in the same configuration, and the one associated with the target was the same for all participants (see Fig. 1).

Conflict tests After learning the goal location in the two training trials, the participants were told that, from now on, they would not get to see where the target was, but that it would always be in the same place as it had been before. The conflict tests consisted of two trials; they were the same as the training trials, except that now the participant did not receive any feedback. While the participant was being spun on the chair, the experimenter moved the set of landmark cards. In one conflict trial, the landmark array was rotated clockwise by $90^{\circ}$, and in the other trial, it was rotated counterclockwise by $90^{\circ}$ relative to its initial position. The configuration of the cards was preserved - the configuration was rotated as a whole array. The end result was that, in one conflict trial, the landmark card associated with the goal was 
displaced horizontally relative to the slope (in a corner with the same elevation, but different on the left-right axis), and in the other trial it was displaced vertically (different elevation, but same position on the left-right axis). Participants allotted the CPs and made the discrete choice in the same way as in the training trials. The facing orientations varied and were counterbalanced across sexes. Also, the trial order was counterbalanced, with half of the sample ( 20 female and 20 male participants) receiving first a horizontal displacement trial (for convenience, referred to as regular order), and the other half receiving first a vertical displacement trial (for convenience, referred to as reverse order). The purpose of these trials was to create a conflict between the two sources of spatial information learned during the training trials; now, the landmarks and the slope information dictated two different goal locations, and on the basis of the CPs allotted and the discrete choices, the preferred reorientation strategies of the participants could be inferred.

Slope test After the conflict trials, one slope test trial was carried out. This followed the same procedure as a conflict trial, except that now the landmark cards were not present (the experimenter removed them while the participant was being disoriented). The purpose of this test was to dissociate slope from the landmark cues; here, only slope information was available, allowing for an assessment of whether the participants encoded a slope-based representation of the goal at all. After this trial, the real-world portion of the experiment was completed, and the participant left the enclosure.

Paper-and-pencil tests In order to examine the correlates of reorientation, in another room each participant took a battery of tests that involved different spatial abilities. These paper tests were always placed flat on the desk. The tests included the following:

1. A spatial-orientation test (SOT; Kozhevnikov \& Hegarty, 2001; we used the revised version created by Hegarty \& Waller, 2004). This test assesses the ability to imagine different orientations or perspectives in space.

2. A water-level test (WLT; Piaget \& Inhelder, 1956; we used the test devised by Liben, 1995). This test assesses the use of the gravity reference frame for inferring the level of a liquid in a tilted bottle.

3. A questionnaire on experience with directional cues, with two questions, each composed of two opposite statements. Participants had to express their agreement with either statement on a scale from 0 (total agreement with the first statement) to 10 (total agreement with the second statement). The first question was composed of these two statements: "Where I grew up, I could find my way based on the slope of the terrain (e.g., going uphill or downhill)," and "Where I grew up, the slope of the terrain was not meaningful to me (or not present at all)." The second question was composed of these two statements: "Where I grew up, I had no idea where North, South, East and West were," and "Where I grew up, I usually knew where North, South, East and West were."

4. A questionnaire on heel use (only for females), in which participants had to express how often they wore heeled footwear (on a scale from 1 to 7) and to give percentages of low-, medium-, and high-heel use.

5. A landmark test, relative to the real-world task carried out in the tilted enclosure. On a sheet of paper was represented the square experimental enclosure, with four bowls and the landmark array in the corners, just as in Fig. 1 (same configuration of cards as in the realworld task, and in an identical rotation for all participants); no information on the direction of the slope gradient relative to the enclosure was provided. On the basis of the landmark cues, the participant had to write down where the target had been hidden during the reorientation task and had to allot CPs and make a discrete choice, just like during the real-world task. This landmark test dissociated landmark cues from slope, and therefore it could be used to infer whether the participant encoded a landmark-based representation of the goal during the reorientation task. As this test was complementary to the slope test, these two tests are referred to as single-cue tests.

One participant's paper-and-pencil tests (all except the landmark test) were lost, so they are not taken into account in any analysis.

Summary of procedure The real-world tasks consisted of training (two trials), the conflict tests (two trials), and the slope test (one trial). The paper-and-pencil tasks consisted of the spatial orientation test, water-level test, questionnaire on experience with directions, questionnaire on heel use, and landmark test. (The slope and landmark tests are referred to as single-cue tests.) The experimental session lasted 45 $50 \mathrm{~min}$ from initial instruction to debriefing; the time elapsed between the first trial (training) and the landmark test was approximately $35 \mathrm{~min}$.

\section{Results}

Training

Performance in the training trials was analyzed with a twofactor mixed ANOVA, with CPs as the dependent variable, Training Trials as the within-subjects factor, and Sex as a between-subjects factor. CPs for the correct hiding place 
increased only marginally from Trial 1 to Trial $2, F(1,78)=$ $3.280, M S E=5.152, p=.074, \eta_{\mathrm{p}}{ }^{2}=.040$. The men allotted significantly more CPs to the correct corner than did the women, $F(1,78)=4.742, M S E=20.594, p=.032, \eta_{\mathrm{p}}{ }^{2}=$ .057 , and we found no sex-by-trial interaction, $F(1,78)=$ $0.098, p=.755, \eta_{\mathrm{p}}{ }^{2}=.001$ (see Fig. 2).

In the first trial, $72.5 \%$ of the participants chose the correct hiding place, and in the second trial, $77.5 \%$ chose it; this difference was not significant, $\chi^{2}(1, n=160)=0.53$, $p=.465$. Breaking down the sample by sex, the frequencies of correct choices were not significantly different between men and women for either Trial $1, \chi^{2}(1, n=80)=1.00, p=$ .317 , or Trial $2, \chi^{2}(1, n=80)=2.58, p=.108$ (see Fig. 2). Overall, the number of correct choices made during training $(0,1$, or 2$)$ did not differ between the sexes, $t(78)=1.59$, $p=.117, d=0.35$.

Dividing the sample on the basis of the strategy used during the conflict tests (see below), the frequency of correct choices was significantly above chance (25\% correct) for all strategy groups (binomial test, $p \mathrm{~s}<.001$ ). However, the numbers of correct choices during training were significantly different among the strategy groups, $F(2,77)=7.80$, $M S E=.432, p<.001, \eta_{\mathrm{p}}{ }^{2}=.169$. The neither-strategy group chose the correct corner significantly less than did either the slope-strategy (Sidak, $p<.05$ ) or the landmarkstrategy (Sidak, $p<.01$ ) group. There was no significant difference between the landmark- and slope-strategy group (Sidak, $p=.182$ ). Similarly, considering the CPs allotted to the correct corner during training, there was a significant difference among the strategy groups, $F(2,77)=13.962$, $M S E=16.240, p<.001, \eta_{\mathrm{p}}{ }^{2}=.266$. The CPs allotted to the correct corner were not significantly different between the landmark-strategy and slope-strategy groups (Sidak, $p=$ .280), but for both groups the CPs allotted to the correct corner were significantly higher than the CPs allotted by the neither-strategy group (Sidak, $p s<.01$ ). The interaction between training trial and strategy group was not significant, $F(2,77)=1.721, M S E=5.002, p=.186, \eta_{p}{ }^{2}=.043$.
Conflict tests

Strategy preference On the basis of their discrete choices during the conflict trials, participants were categorized into three groups. If they consistently chose the slope-correct location on both trials, they were considered to be in the "slopestrategy" group; if they consistently chose the landmarkcorrect location, they were considered to be in the "landmark-strategy" group; if they did not consistently choose a location that was correct according to a type of cue, they were considered to be in the "neither-strategy" group. The frequencies of participants in these categories are shown in Fig. 3, and these proportions do not deviate significantly from a homogeneous distribution, $\chi^{2}(2, n=80)=0.475, p=.789$, suggesting that one cue did not guide reorientation for everyone, but that the two strategies were similarly accessible. Furthermore, the frequency distributions were not significantly different between men and women, $\chi^{2}(2, n=80)=2.84, p=.241$, and, when breaking down the sample by conflict trial order (regular - i.e., first a horizontal displacement trial — or reverse -i.e., first a vertical displacement trial), the difference between distributions of strategy preferences was not significant, $\chi^{2}(2, n=80)=4.52, p=.105$.

Considering the neither-strategy group, out of the 24 participants who fell into this category, only six of the participants chose a correct corner in both conflict trials (oscillating between the slope-correct corner in one trial and the landmarkcorrect in the other); most of the participants (14) chose a correct corner in just one of the conflict trials, and the remaining ones (four) did not choose a correct location in either trial. Considering the discrete choices to a correct corner in both conflict trials, we did not find a significant preference of the neither-strategy group for the slope-correct (11 choices out of 48 ) or the landmark-correct (15 out of 48) corner (binomial test, $p=.557$ ). The overall frequencies of participants who consistently chose one strategy, who oscillated between the two strategies, or who at least once chose an incorrect corner are represented in Fig. 4.
Fig. 2 Performance during the two training trials. On the lefthand side, the mean confidence points (CPs) allotted to the correct corner are shown $( \pm 1 S D)$; men were significantly more confident about the goal location than were women. On the right-hand side, the percentages of participants making a discrete choice to the correct corner are shown; the difference between sexes is not significant

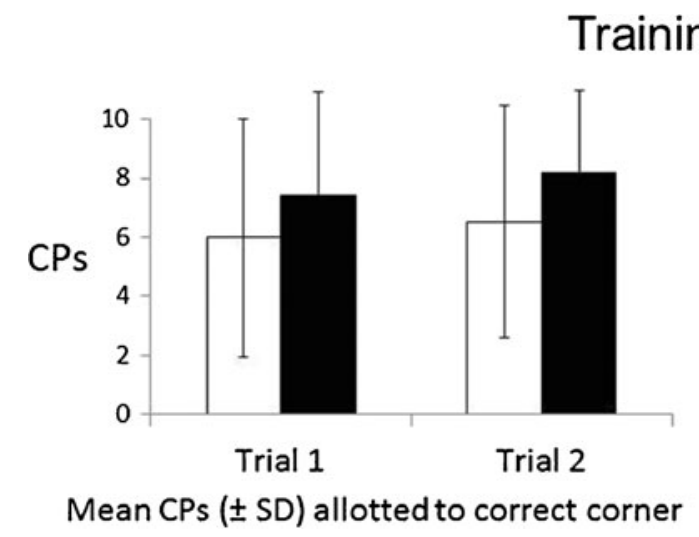

\section{Training trials}

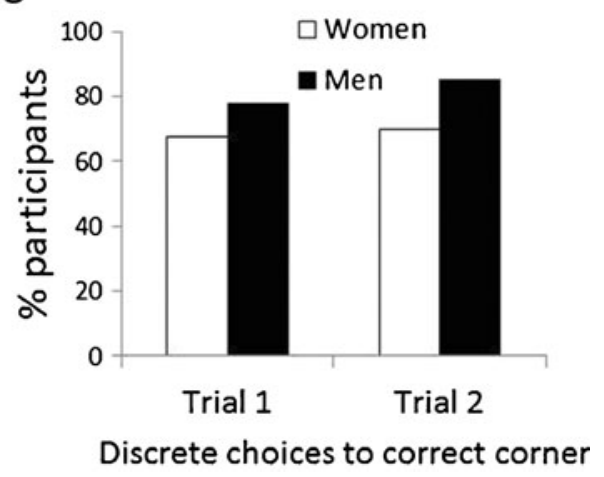




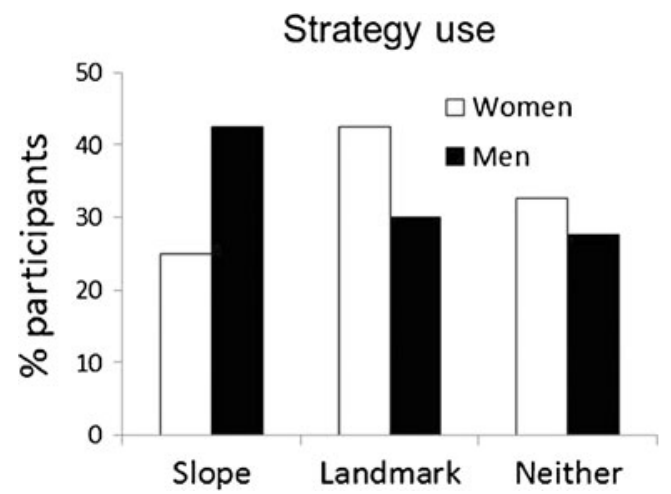

Fig. 3 Strategy use during the two conflict trials: Percentages of participants making discrete choices consistently to the slope-correct corner ("slope strategy"), consistently to the landmark-correct corner ("landmark strategy"), or not consistently choosing a corner that was correct according to any type of cue ("neither strategy"). There was no significant difference between sexes in the distributions of strategy use

In order to specifically compare men's and women's reliance on slope, we considered for each participant the number of slope-correct choices made in the conflict trials $(0,1$, or 2$)$; these were not significantly different between the sexes, $t(78)=1.10, p=.274, d=0.25$. Furthermore, cue reliance was not significantly different between the sexes if we considered the number of landmark-correct choices, $t$ (78) $=0.87, p=.389, d=0.19$.

If, instead of considering the strategy preference per participant, we took into account each and every discrete choice separately for the two conflict trials (a total of 160 choices) and coded them as slope-correct, landmark-correct, or incorrect choice, the frequency of incorrect choices (22 out of 160) was significantly less than the frequencies of either slope-correct (65 out of 160 ; binomial test, $p<.001$ ) or landmark-correct (73 out of 160; binomial test, $p<.001$ ) choices, whereas there was no significant difference between the frequencies of slope-correct and landmarkcorrect choices (binomial test, $p=.551$ ). This suggests, again, that the two strategies were used in similar proportions. Furthermore, there was no significant difference in strategy preference distributions when comparing the sexes, $\chi^{2}(2, n=160)=2.10, p=.350$, or trial order groups, $\chi^{2}(2, n=160)=3.84, p=.147$.

Cue weighting Considering the CPs allotted in the conflict trials, $55.0 \%$ of participants in the first trial, and $60.0 \%$ in the second trial, allotted all of their CPs (ten out of ten) to just one correct corner (whether slope-correct or landmark-correct). In contrast, CPs were broken down between the two correct corners by only $12.5 \%$ of the participants in the first conflict trial, and by $10.0 \%$ in the second conflict trial. The remaining participants $(32.5 \%$ in the first trial and $30.0 \%$ in the second trial) allotted at least some CPs to an incorrect corner. These frequency distributions are represented in Fig. 4, averaged between the two conflict trials.

Considering the whole sample, there was not a significant difference between the CPs allotted to the slope-correct and landmark-correct corners [first conflict trial, $t(79)=1.26, p=$ $.211, d=0.26$; second conflict trial, $t(79)=0.43, p=.670, d=$ 0.09]; however, each strategy group allotted the preponderance of CPs to the corner associated with the strategy that they were using. Participants in the landmark-strategy group allotted, in both conflict trials, $95.8 \%$ of their cumulative CPs to the landmark-correct corner, and only $1.1 \%$ to the other correct corner (slope-correct). Similarly, participants in the slope-strategy group allotted $86.6 \%$ of their cumulative CPs to the slope-correct corner, and only $6.2 \%$ to the other correct corner (landmark-correct). In contrast, the neither-strategy group divided their cumulative CPs approximately equally among the correct corners $(51.4 \%)$ and the incorrect ones (48.6\%; binomial test, $p=.553$ ); their cumulative CPs were also divided approximately equally between the slope-correct $(24.9 \%)$ and landmark-correct $(26.5 \%)$ corners (binomial test, $p=.655$ ).

\section{Cue weighting}

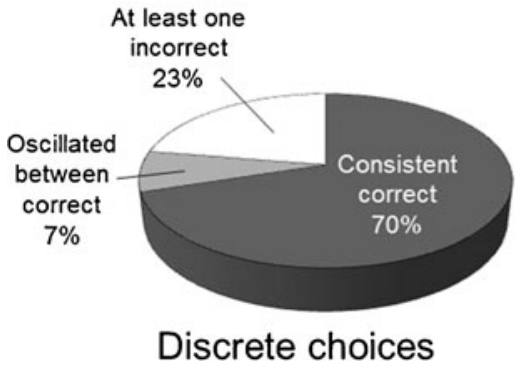

Fig. 4 Weighting of slope and landmark cues during the two conflict trials. On the left-hand side, the percentages of participants making discrete choices consistently to the same type of correct corner (in either slope- or landmark-correct trials), oscillating between the two types of correct corners (in one trial, slope-correct, and in the other, landmark-correct), or choosing at least once an incorrect corner. On the

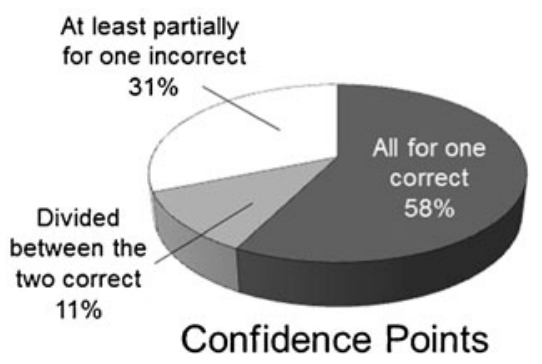

right-hand side are the percentages of participants who allotted the whole set of CPs to just one correct corner (slope- or landmarkcorrect), who divided their CPs between the two correct corners, or who allotted at least some CPs to an incorrect corner (the data are averaged throughout the two conflict trials) 
Again, in order to compare strategy use between the sexes, we analyzed the CPs allotted to the slope-correct corner during the conflict trials. There was no significant difference between trials $\left[F(1,76)=0.226, M S E=4.329, p=.636, \eta_{\mathrm{p}}{ }^{2}=.003\right]$, no significant effect of order [regular or reverse; $F(1,76)=1.963$, $\left.M S E=30.267, p=.165, \eta_{\mathrm{p}}{ }^{2}=.025\right]$, and no significant effect of $\operatorname{sex}\left[F(1,76)=2.521, p=.116, \eta_{\mathrm{p}}{ }^{2}=.032\right]$; furthermore, the interactions were not significant $(p s>.116)$. The same result emerged if we analyzed the CPs allotted to the landmarkcorrect corner: There were no significant differences between trials $[F(1,76)=0.353, M S E=5.733, p=$ $\left..554, \eta_{\mathrm{p}}^{2}=.005\right]$, no significant effects of order $[F(1$, 76) $\left.=0.669, M S E=35.933, p=.416, \eta_{\mathrm{p}}{ }^{2}=.009\right]$ and $\operatorname{sex}\left[F(1,76)=0.507, p=.479, \eta_{\mathrm{p}}{ }^{2}=.007\right]$, and no significant interactions $(p \mathrm{~s}>.325)$.

\section{Single-cue tests}

Performance in the two single-cue tests was compared using a 2 (men vs. women) by 2 (slope test vs. landmark test) mixed ANOVA on the CPs allotted to the correct corner (Fig. 5). There was no significant effect of test $[F(1,78)=$ 2.41, MSE $\left.=18.52, p=.125, \eta_{\mathrm{p}}{ }^{2}=.030\right]$, suggesting that the two cues were not encoded differently; furthermore, there was no effect of sex $[F(1,78)=0.02, M S E=13.14, p=$ $\left..888, \eta_{\mathrm{p}}{ }^{2}=.000\right]$, indicating that men and women did not have different cue encoding preferences; finally, the sex-bytest interaction was also not significant, $F(1,78)=2.30, p=$
$.133, \eta_{\mathrm{p}}{ }^{2}=.029$. The correlation between the CPs allotted to the correct corners in the slope and landmark tests was not significant, considering the overall sample, $r(78)=-.184$, $p=.102$. However, when considering only participants who clearly were following a strategy (i.e., excluding the neitherstrategy group), we did find a significant negative correlation between CPs allotted to the correct corner in the two single-cue tests, $r(54)=-.534, p<.001$; this suggests that the more confident a participant was about the encoding of one cue, the less confident he or she was about the other. This significance holds even when controlling for sex, $r(53)=-.511, p<.001$.

When analyzing specifically the CPs allotted to the correct corner in the slope test (Fig. 5), there was a significant effect of order, $F(1,76)=7.660, M S E=12.781, p=.007, \eta_{\mathrm{p}}{ }^{2}=.092$, and the effect of sex was not significant, $F(1,76)=1.937, p=$ $.168, \eta_{\mathrm{p}}{ }^{2}=.025$; however, the sex-by-order interaction was significant, $F(1,76)=4.861, p=.030, \eta_{\mathrm{p}}^{2}=.060$, and therefore we analyzed the simple effects. Male participants allotted significantly more CPs to the slope-correct corner in the reverse group (with a vertical-displacement conflict trial first) than in the regular order group (with a horizontal-displacement trial first; $p=.001, \eta_{\mathrm{p}}{ }^{2}=.140$ ); the order of the conflict trials (regular or reverse) did not affect the performance in the slope test for female participants $\left(p=.692, \eta_{\mathrm{p}}^{2}=.002\right)$.

The CPs allotted to the correct corner in the landmark test were not significantly affected by order $[F(1,76)=0.035$,
Fig. 5 Results of the single-cue tests. The top left graph shows the mean CPs $( \pm 1 S D)$ allotted to the correct corners in the slope test and the landmark test; there was not a significant difference between sexes in either test, nor a significant sex-bytest interaction. The top right graph shows the percentages of participants making a correct discrete choice in the slope test and the landmark test; the frequencies were not significantly different between the sexes. The bottom graph shows the percentages of participants making a correct discrete choice in both tests, in only the slope test, in only the landmark test, or in neither test; there was not a significant difference between sexes in the distribution. Note that, while the slope test was a real-world task, the landmark test was a paper version of the task

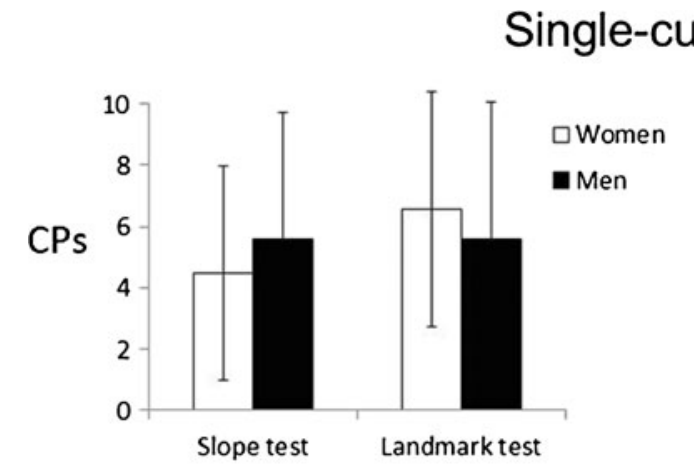

Mean CPs ( \pm SD) allotted to correct corner

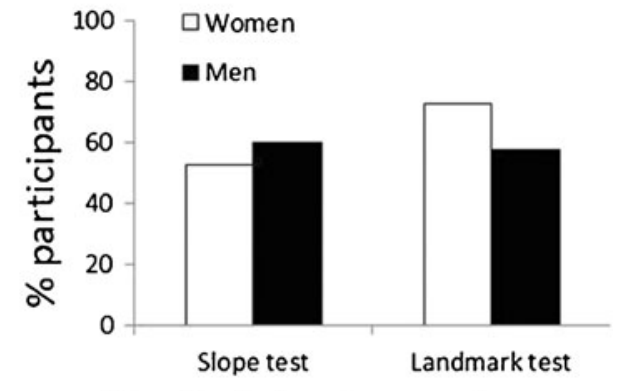

Discrete choices to correct corner

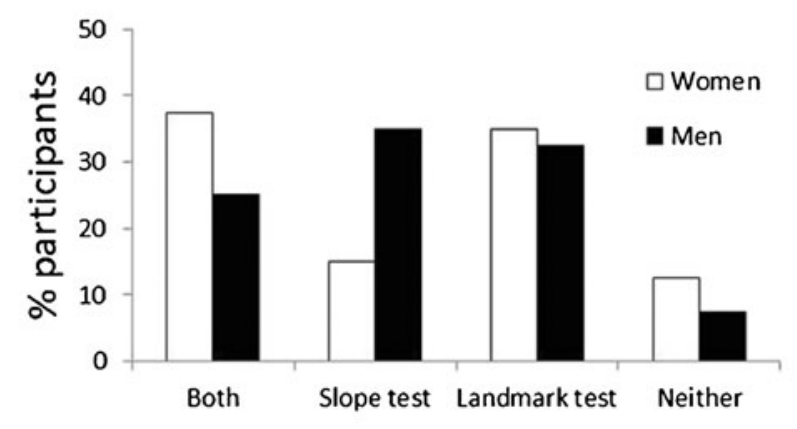

Discrete choices to correct corner 
$\left.M S E=17.563, p=.852, \eta_{\mathrm{p}}{ }^{2}=.000\right]$ or $\operatorname{sex}[F(1,76)=1.028$, $\left.p=.314, \eta_{\mathrm{p}}{ }^{2}=.013\right]$, and the sex-by-order interaction was not significant $\left[F(1,76)=0.139, p=.710, \eta_{\mathrm{p}}^{2}=.002\right]$ (Fig. 5).

Discrete choices in the single-cue tests are also shown in Fig. 5, and they reveal no significant sex differences in the encoding of the slope and landmark cues. The distributions of correct choices in the two single-cue tests were not significantly different between men and women, $\chi^{2}(1, n=$ $97)=0.800, p=.371$. Furthermore, in the slope test, the frequencies of participants who chose the correct corner did not significantly differ between sexes, $\chi^{2}(1, n=80)=0.457$, $p=.499$, or between conflict trial order groups, $\chi^{2}(1, n=$ $80)=2.489, p=.115$. In the same way, in the landmark test, the frequencies of participants who chose the correct corner did not significantly differ between sexes, $\chi^{2}(1, n=80)=$ $1.978, p=.160$, or between conflict trial order groups, $\chi^{2}(1$, $n=80)=0.0, p=1$. The frequencies of correct choices in each test were significantly above chance for both male and female participants (binomial test, $p \mathrm{~s}<.001$ ). Finally, considering the distribution of participants who chose the correct corner in the slope test only, in the landmark test only, in both tests, or in neither test, we also found no significant differences between the sexes, $\chi^{2}(3, n=80)=4.737, p=$ .192 , or between conflict trial order groups, $\chi^{2}(3, n=80)=$ $3.493, p=.322$.

\section{Correlates of performance}

In order to better understand the individual differences in reorientation, we looked into the correlates of performance. Because, as mentioned above, there were no significant differences between the CPs allotted to the correct locations in the two training trials, an aggregated value was used for each participant (the sum of the CPs for the two training trials). In the same way, an aggregated value was used to capture confidence for the slope-correct and the landmarkcorrect corners in the conflict trials (the sum of the CPs allotted to the slope-correct and landmark-correct corners, respectively, in the two conflict trials). These correlations are summarized in Fig. 6. Performance in the reorientation task, as measured by CPs allotted to the correct corner in the training trials, correlated significantly with CPs allotted to the landmark-correct corner during the conflict trials $[r(78)=$ $.395, p<.001]$; this indicates that, in general, participants who were more confident in solving the task were more certain about the target location based on landmark cues (in other words, they were more confident in relying on the landmark strategy). Furthermore, performance in the task correlated with CPs allotted to the correct corner in both single-cue tests [slope test, $r(78)=.243, p=.030$; landmark test, $r(78)=.508, p<.001]$; this indicates that better performers were more confident in locating the target when either cue was presented in isolation.
Considering the paper-and-pencil tests, the WLT was not significantly different between sexes, $t(77)=1.23, p=.223$, $d=0.28$, whereas in the SOT, men were significantly more accurate than women, $t(77)=2.25, p=.028, d=0.50$. The questionnaire on directional experience did not reveal a significant sex difference relative to Question 1, $t(77)=$ $1.59, p=.116, d=0.36$, and revealed only a marginally significant difference for Question 2, $t(77)=1.74, p=.087$, $d=0.39$ (men agreed marginally more than women with the statement "Where I grew up, I usually knew where North, South, East and West were").

Both the WLT $[r(77)=.259, p=.021]$ and the SOT $[r(77)=$ $-.450, p<.001]$ correlated significantly with performance in the reorientation task (training trials). Furthermore, the WLT correlated with CPs allotted to the slope-correct corner in the conflict trials $[r(77)=.236, p=.037]$, and with the CPs allotted to the correct corner in the slope test $[r(77)=.298, p=.008]$; in contrast, the SOT correlated with CPs allotted to the correct corner in the landmark test $[r(77)=-.405, p<.001]$.

Neither the questionnaire on heel use nor the one on directional experience correlated with any measure in the experiment.

\section{Discussion}

This study was designed to examine how people reorient in a sloped environment when they also have the possibility of using landmark cues. We used the same apparatus and inclination as Nardi et al. (2011), except for one crucial difference: A set of distinct objects were provided, which could be used as landmarks to encode the target location. Therefore, for the first time in a real-world experiment, men and women could use either a slope strategy or a landmark strategy to solve a reorientation task. This addition made the task easier to solve; in fact, comparing the two training trials of the present experiment with the first two training trials of Nardi et al. (2011, Exp. 1), the frequency of correct choices was significantly higher in the present experiment, increasing from $55 \%$ to $75 \%$ (Fisher's exact test, $p<.01$ ). This suggests that, even though we used a set of cards with fairly subtle differences (patterns of black and white), these objects were salient enough to be successfully used as landmarks, improving overall reorientation performance considerably relative to a basic, impoverished environment with the slope of the floor as the only informative cue (as in Nardi et al., 2011). However, as is shown in Fig. 3, the sample as a whole chose the slope-based and landmark-based strategies with approximately equal frequencies, with about one third of the participants in each strategy group. The remaining third of the sample fell into the neither-strategy group category, because they did not show consistent use of a single cue. This indicates that, in general, one type of information 


\section{CORRELATIONS}

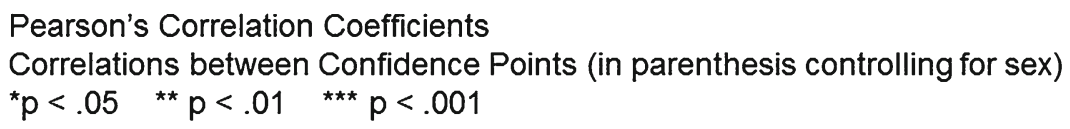

\begin{tabular}{|c|c|c|c|c|}
\hline & \multicolumn{2}{|c|}{ Reliance on: } & \multicolumn{2}{|c|}{ Encoding of: } \\
\hline & Slope & Landmark & Slope & Landmark \\
\hline Reorientation & $\begin{array}{c}.052 \\
(.010)\end{array}$ & $\begin{array}{l}.395 * * * \\
(.428) * * *\end{array}$ & $\begin{array}{l}.243 * \\
(.217)\end{array}$ & $\begin{array}{l}.508 * * * \\
(.556) * * *\end{array}$ \\
\hline
\end{tabular}

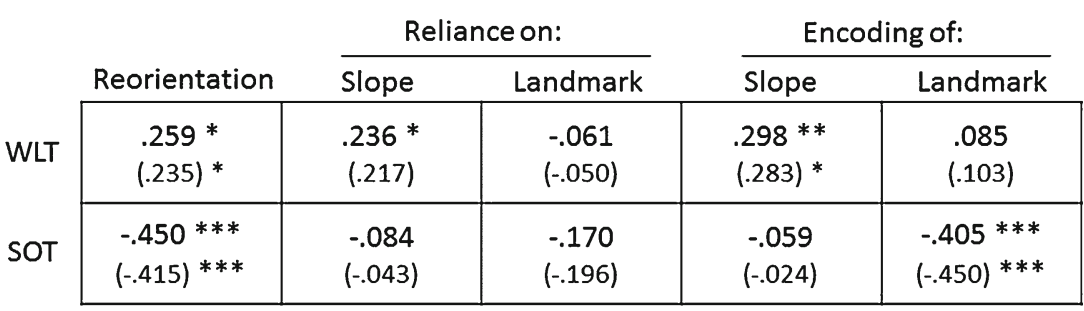

WLT $=$ Water Level Test $\quad$ SOT $=$ Spatial Orientation Test

Fig. 6 Correlation tables. The Reorientation factor denotes CPs allotted to the correct corner during the two training trials (aggregated across both trials). Reliance on Slope or Landmark denotes CPs allotted to the slope-correct or the landmark-correct corner, respectively, during the two conflict trials (aggregated across both trials). Encoding of Slope and Landmark denotes CPs allotted to the correct corner during the slope and landmark tests, respectively. The top table shows correlations between these factors. In the bottom table, the water-level

did not dominate the reorientation process for everyone: Stimulus salience was balanced between slope and landmarks, and both types of cues enabled similarly accessible strategies.

During the experiment, participants searched for a target, making a discrete choice and allotting ten confidence points (CPs) among the potential hiding locations. The use of a graded measure of task performance provided us with more information on the cognitive process of reorientation. We focus our discussion below on three main issues.

\section{Cue weighting}

By adding landmarks to the slope of the environment, a new level of complexity was introduced in the task: Which cue would be used to reorient and locate the target, and how would the cues be weighted in the process? Research has addressed this type of question in the case of a continuous search space, and it has been proposed that cues are combined and weighted optimally on the basis of their variance, according to a Bayesian model (Cheng, Shettleworth, Huttenlocher, \& Rieser, 2007; Nardini, Jones, Bedford \& Braddick, 2008). But it is also important to understand how cues are weighted in discrete search spaces, because in everyday life we often have to decide between here or there. test (WLT) and the spatial-orientation test (SOT) were paper-andpencil tests on spatial abilities. The WLT assessed the use of the gravity reference frame for inferring the level of a liquid in a tilted bottle (the higher the score, the greater the use of the gravity reference frame), and the SOT assessed the ability to imagine different orientations or perspectives among objects in space (the higher the score, the larger the error)

The vast majority of the literature using discrete hiding locations has also employed discrete choices as the dependent variable (a location can either be chosen or not), which made it very hard to estimate the degree to which participants rely on one cue as compared to another. Therefore, in the present study, we were very interested in assessing how participants would allot their ten CPs when the slope and landmark cues predicted different target locations in the conflict trials: Would participants show a combination of strategies - spreading the CPs between two correct corners - or would they univocally rely on one-allotting all of their CPs to just one correct corner?

The average proportion of participants who, in a situations of conflict, were fully confident (allotting ten CPs out of ten) in just one type of correct corner (either slope- or landmark-correct) was more than five times larger than the proportion of those who spread their confidence between the two types of correct corners ( $57.5 \%$ vs. $11.3 \%)\left[\chi^{2}(1, n=\right.$ $80)=37.929, p<.001$; see Fig. 4]. Furthermore, excluding participants who were not clearly using a strategy (i.e., the neither-strategy group), the vast majority of CPs were allotted to the chosen corner (slope- or landmark-correct), and only very few to the other correct corner. These data fit a scenario in which participants, in order to solve the reorientation task, were strongly relying on just one strategy rather 
than taking into account both types of information. The neither-strategy group behaved differently: They divided their cumulative CPs approximately equally between the correct and incorrect corners, suggesting that they were not confused by having to choose between the two possibly correct locations - they were actually lost and allotted CPs at random. Failure of the neither-strategy group to use the available spatial information is probably why they exhibited lower confidence and poorer performance during the training trials.

Although this analysis is admittedly descriptive, the hypothesis of univocal reliance on one source of information for reorientation nicely fits with evidence from the singlecue tests. From Fig. 5, one can see that the proportion of participants who encoded both cues (31.3\%) is significantly smaller (almost by half) than the combined proportion of participants who encoded only a single cue $(58.8 \%)\left[\chi^{2}(1\right.$, $n=80)=12.222, p<.001]$. Even more intriguing is the fact that, excluding the neither-strategy group, confidence for the correct corner was negatively correlated between the two single-cue tests; in other words, the more a participant was confident about one cue, the less confidence was exhibited about the other cue. These findings do not fit with a scenario in which participants encode all of the available spatial information; they are consistent with a scenario in which participants encode the least amount of information (sufficient for a single strategy) necessary to solve the task. This hypothesis is in apparent contrast with animal research, which shows, in general, that if two cues are available for solving a reorientation task, the subjects clearly encode both (for birds, see Vargas, Petruso \& Bingman, 2004b; for fish, Vargas, Lopez, \& Thinus-Blanc, 2004a; for rats, Cheng, 1986; for monkeys, Gouteux, Thinus-Blanc, \& Vauclair, 2001; for a review, see Cheng \& Newcombe, 2005). However, it is important to note that most of these studies were based on long training sessions, with the total number of training trials on the order of tens. After receiving all of this training, animals are probably at the ceiling of spatial learning and have had enough exposure to the environment to encode redundant cues. This was not the case in our task, as participants only had two trials to learn the cues associated with the target location. In this condition, it makes intuitive sense that participants did not have time to fully encode the environment, and paid attention to just one cue. This cognitively parsimonious model of spatial learning is suitable for acquiring a task as quickly and effectively as possible, and might apply also to more ecologically relevant spatial tasks (e.g., trying to remember one's way back to a place in a novel environment).

As a final note, it is possible that if, instead of the CPs, we had used a more cognitively implicit dependent variable - a measure that required less conscious awareness of problem-solving - it would have revealed a different pattern of cue weighting. However, if we consider the discrete choices made during the conflict trials (Fig. 4), $70.0 \%$ of the participants consistently selected the corner associated with one cue, and only $7.5 \%$ chose the corner associated with slope in one trial and landmark in the other trial. Therefore, this behavioral measure of reorientation shows no sign of oscillation between the two potentially correct locations, as would be expected with a strategy that used a combination of the cues (Cheng \& Newcombe, 2005). Participants overwhelmingly stuck with one cue.

\section{Correlates of reorientation}

During the training trials, $41 \%$ of the participants were maximally confident (ten CPs out of ten) about the location of the hidden target in both trials; the rest showed some degree of uncertainty, with $36 \%$ allotting fewer than five CPs to the correct corner in at least one trial, and $5 \%$ being completely unconfident about the goal location (zero CPs out of ten in both trials). What are the reasons for these individual differences?

Performance on the reorientation task, as measured by CPs for the target location during the training trials, correlated with the four following factors.

1. Sex. Men were significantly more confident than females about the correct hiding place. We will address this point later, when we specifically deal with sex differences.

2. Performance on the WLT and SOT. Even though smallscale and large-scale spatial abilities are partially dissociated, the shared variance between these psychometric tests and real-world reorientation confirms an underlying factor, based on the ability to encode, use, and manipulate spatial information (Hegarty, Montello, Richardson, Ishikawa, \& Lovelace, 2006). As a side note, the WLT and the SOT seem to be associated with the encoding of different cues in our experiment (see Fig. 6). In the single-cue tests, slope and landmarks were presented in isolation, one at a time; the CPs allotted to the corner predicted by the available cue indicates, therefore, the extent to which participants encoded that cue and could use it to locate the target. Performance on the WLT correlated with the CPs allotted to the correct corner in the slope test; the link between the WLT and slope use was identified in our previous study (Nardi et al., 2011), and suggests an ability to use the vertical/gravity reference frame. In contrast, the SOT correlated with the landmark test, and this is probably related to an ability to reorient among an array of objects.

3. Confidence for the correct corner in both single-cue tests. This correlation confirms a predictable link between reorientation and encoding of the cues that support it. 
4. Confidence for the landmark-correct corner in the conflict tests. In these trials, landmark and slope information determined two different correct target locations, and the CPs allotted among the corners revealed reliance on the landmark strategy (CPs allotted to the landmark-correct corner), the slope strategy (CPs allotted to the slope-correct corner), or neither strategy (CPs allotted to incorrect corners). Therefore, posing the correlation in other terms, participant who weighted more heavily the landmark strategy were also more confident in solving the reorientation task; on the contrary, those relying more heavily on the slope strategy were not necessarily more confident in the task. Therefore, at least in a relatively small environment, the landmarks employed in our experiment seemed to sustain a more effective and confident reorientation process. This could be related to the nature of landmarks as a reference frame. In our task, in order to locate the target using landmarks, it would have sufficed to learn an association between a specific card and the target (not necessarily the card at the correct corner; e.g., "the target is in the corner opposite that card"). This might be a comparatively simpler process than using the slope gradient. In a square, tilted environment like the one that we used, research has shown that a goal location is represented with a mixed reference frame: The target is mainly encoded as uphill or downhill (allocentric), and the left-right coordinate is encoded egocentrically relative to the participant's body (e.g., facing downhill, the goal is on my left; Nardi et al., 2011; Weisberg, Nardi, Newcombe, \& Shipley, 2012). The hypothesis of landmarks being a more helpful cue is also supported by previous research on directional (including slope) versus positional (landmark) cues within a virtual environment (VE). When training with only one type of cue, landmarks lead to more accurate goal searching, as compared to directional cues (Chai \& Jacobs, 2009); furthermore, after training with both types of cues, removing positional cues causes a significant decrease in searching efficiency, whereas removing directional cues does not (Chai \& Jacobs, 2010). Taken together, this evidence argues for landmark strategies giving a stronger contribution to reorientation and goalsearching performance - at least in a small-scale environment - as compared to strategies based on directional cues (including terrain slope).

\section{Sex differences}

With a relatively large sample size and by analyzing both discrete choices and $\mathrm{CPs}$ - which provide a more graded measure of behavior - we identified evidence in support of sex differences in only two areas. One was in the slope test, in which men were affected by conflict trial order (regular vs. inverse), but women were not. During the conflict trials, in a vertical displacement the correct landmark/card would assume a different position along the vertical axis of the slope gradient (an opposite elevation; if it used to be uphill, now it would be downhill); because the vertical axis of a slope is more salient than the horizontal (J. W. Kelly, 2011; Nardi et al., 2011), a vertical displacement causes a larger conflict than does a horizontal displacement. Receiving the larger conflict situation first in the conflict trials (inverse order) might have increased the participants' awareness of the slope to a greater degree than receiving the larger conflict second (regular order); this higher slope awareness could have determined the greater confidence for the correct corner when only slope was available (in the slope test). The fact that only men showed this effect suggests that the sexes had different sensitivities to conflict situations.

More interesting is the finding that in the reorientation task (training trials), men allotted significantly more CPs to the correct location. This disparity, however, was attenuated in the discrete choices. Considering both the trial-by-trial analyses and the overall number of correct choices during training, sex differences were not significant, although they trended in the same direction. In comparison, in our previous study (Nardi et al., 2011), women showed significantly less accurate discrete choices after only two training trials. Therefore, in the present experiment it seems that women, rather than underperforming, showed more uncertainty when solving the task. The literature on spatial abilities abounds with reports of sex differences in performance, most of which are in favor of males (D. M. Kelly \& Bischof, 2005; Moffat, Hampson, \& Hatzipantelis, 1998; Sandstrom, Kaufman, \& Huettel, 1998; Saucier et al., 2002; Schinazi et al., 2009; Voyer et al., 1995). However, women have also been shown to display inferior spatial confidence (in a cognitive mapping task, O'Laughlin \& Brubaker, 1998; in way-finding, Lawton, Charleston, \& Zieles, 1996; in distance estimation, Foley \& Cohen, 1984), lower self-reported spatial skills (e.g., SBSOD; Hegarty et al., 2006), as well as greater levels of spatial anxiety (Lawton, 1994; Lawton \& Kallai, 2002). Importantly, factors relating to spatial confidence can alter spatial behavior, which, depending on the variable used in the task, may lead to sex differences in performance (Lavenex \& Lavenex, 2010; Lawton et al., 1996). For example, a lack of confidence can be associated with increased exploratory behavior, possibly causing longer latencies and perseveration errors (Lavenex \& Lavenex, 2010). To the best of our knowledge, the present study is the first to show evidence of inferior confidence for women in reorientation, reinforcing the view of spatial confidence as a sex-typed quality dissociable from spatial ability. 
Except for these differences, men and women behaved similarly when we took into account both the discrete choices and CPs allotted in the conflict tests and the single-cue tests. Importantly, we did not find convincing evidence in support of different reliance on slope or landmark cues. Considering discrete choices, the frequency of participants in each strategy group differed between sexes by only seven participants in the slope-strategy group, and by five participants in the landmarkstrategy group (see Fig. 3); furthermore, the numbers of slopecorrect choices (or landmark-correct choices) during the conflict trials were not significantly different between men and women. Moreover, when considering the CPs allotted to the slope-correct or landmark-correct corners, there was not a significant difference between sexes. The lack of significantly different cue preferences extended to the single-cue tests: Men and women did not show a sex-specific encoding of cuesrelative to either discrete choices or CPs (see Fig. 5). It should be noted that, in all of these analyses, the data trended toward slight, nonsignificant, but consistent preferential uses of slope by men and of landmarks by women. Similar strategy differences, with men relying more on directional cues (mainly terrain slope) than do women, have been found to be statistically significant when using a VE (Chai \& Jacobs, 2009, 2010). In this regard, it is important to note that these studies used sample sizes comparable to ours (84 participants in Chai \& Jacobs, 2009; 51 participants in Chai \& Jacobs, 2010). Furthermore, we estimated from the published results that the effect sizes found - according to Cohen's (1988) convention-ranged from "medium" (Cohen's $f=.28$ for Chai \& Jacobs, 2009) to "large" (Cohen's $w=.49$ for Chai \& Jacobs, 2010). For our sample size, the statistical powers to detect similar-sized effects were .6 and .9, respectively (Erdfelder, Faul, \& Buchner, 1996). Therefore, it is unlikely that the lack of a significant strategy difference in our study was due simply to low power. We believe that using a real-terrain slope, which could be walked, increased the salience of the directional cue as compared to a virtual slope presented on a computer monitor, making this cue more accessible to use. It has been shown that the size of computer displays can affect sex differences in virtual navigation: When a smaller display is used and less optic flow is provided, the female disadvantage is significantly increased (Tan, Czerwinski, \& Robertson, 2006). In the same way, the slope strategy might have been less readily available for the women in the VE studies (Chai \& Jacobs, 2009, 2010) because the sensory information associated with the slope gradient was impoverished (vestibular and kinesthetic cues were not provided). In sum, the sex differences in strategy use found in VE seem to generalize weakly, if at all, to a real environment, or, put in a different way, sex-specific preferences for directional (slope) and positional (landmark) cues seem to be accentuated in virtual as compared to real environments.

\section{Conclusions}

The present study builds on our previous one (Nardi et al., 2011) and has important implications regarding reorientation and slope use. In the previous study, women exhibited difficulty dealing with slope when that was the only cue available for reorientation. As compared to men, performance in the task was worse, and the time required to correctly identify the uphill side of the enclosure was longer. The present study suggests that the female disadvantage is not due to an inherent lower preference for using slope when other effective strategies are available; from a different perspective, it suggests that, even if males have an advantage with slope, when given a choice of cues to use, their preference for slope is not significantly higher as compared to females, and a substantial proportion of men - not significantly different from that of women-prefer to use landmarks. Furthermore, the present study does not support a role of footwear habits. Women are used to wearing heeled shoes more often than men; this might impair the sensitivity to slope as a consequence of reduced perceived foot tilt. In our previous work, we found that the footwear worn at the time of the experiment did not affect women's performance. However, an unanswered question was whether prior experience with heeled footwear had a role. With the questionnaire on heel experience, in the present study, we addressed this issue. The questionnaire did not correlate with any measure in the experiment; in particular, the frequency and the variety of heel heights used did not show any relation with either reliance on or encoding of slope information. This suggests that footwear habits cannot be held accountable for the female difficulty with slope. So, which factors are responsible?

The evidence accumulated to date seems to indicate that the female disadvantage is associated with perceptual/attentional factors, possibly related to lower sensitivity to vestibular information (Sholl, 1989) and to the kinesthetic component of slope perception (Weisberg et al., 2012); in addition, the difficulty could be due to experience (familiarity) with directional cues in general (consistent with our finding from the questionnaire on experience with directional cues, which showed a marginally significant sex difference on one question). Further research will be needed to explore these possible causes. However, the present study highlights another plausible factor: The female difficulty reorienting with only slope could also derive from lower spatial confidence. In Nardi et al. (2011), uncertainty for the target location could have led, over a series of four trials, to more hesitation and an increased amount of errors, deteriorating women's performance; in addition, when having to point to the uphill direction of the slope gradient, lower confidence could have caused women to assume a more conservative criterion of response, resulting in longer 
response times. In the present study, instead, because confidence was recorded separately from discrete choices, females did not suffer inferior performance, but still showed greater uncertainty reorienting. In this respect, it is extremely interesting that women exhibited lower confidence even though a landmark strategy was available — a strategy that is considered simple (Ratliff \& Newcombe, 2008) and, if anything, is supposedly more female-related (Galea \& Kimura, 1993; Jacobs \& Schenk, 2003; Ward, Newcombe, \& Overton, 1986). This suggests that spatial confidence might affect performance in a very wide range of conditions, even when potentially more accessible strategies are available. Future studies should take this into account and examine more extensively the effects of spatial confidence on spatial abilities.

Author note This research was supported by NSF Grant No. SBE0541957 to SILC, for which we are very grateful.

\section{References}

Chai, X. J., \& Jacobs, L. F. (2009). Sex difference in directional cue use in a virtual landscape. Behavioral Neuroscience, 123, 276283

Chai, X. J., \& Jacobs, L. F. (2010). Effects of cue types on sex differences in human spatial memory. Behavioural Brain Research, 208, 336-342.

Cheng, K. (1986). A purely geometric module in the rat's spatial representation. Cognition, 23, 149-178.

Cheng, K., \& Newcombe, N. S. (2005). Is there a geometric module for spatial orientation? Squaring theory and evidence. Psychonomic Bulletin Review, 12, 1-23. doi:10.3758/BF03196346

Cheng, K., Shettleworth, S. J., Huttenlocher, J., \& Rieser, J. J. (2007). Bayesian integration of spatial information. Psychological Bulletin, 133, 625-637.

Cohen, J. (1988). Statistical power analysis for the behavioral sciences (2nd ed.). Hillsdale, NJ, Erlbaum.

Erdfelder, E., Faul, F., \& Buchner, A. (1996). GPOWER: A general power analysis program. Behavior Research Methods, Instruments, \& Computers, 28, 1-11. doi:10.3758/BF03203630

Foley, J. E., \& Cohen, A. J. (1984). Mental mapping of a megastructure. Canadian Journal of Psychology, 38, 440-453.

Galea, L. A., \& Kimura, D. (1993). Sex differences in route-learning. Personality and Individual Differences, 14, 53-65.

Gouteux, S., Thinus-Blanc, C., \& Vauclair, J. (2001). Rhesus monkeys use geometric and nongeometric information during a reorientation task. Journal of Experimental Psychology. General, 130, $505-519$.

Hegarty, M., Montello, D. R., Richardson, A. E., Ishikawa, T., \& Lovelace, K. (2006). Spatial abilities at different scales: Individual differences in aptitude-test performance and spatial-layout learning. Intelligence, 34, 151-176.

Hegarty, M., \& Waller, D. (2004). A dissociation between mental rotation and perspective-taking spatial abilities. Intelligence, 32, 175-191.

Hermer, L., \& Spelke, E. (1994). A geometric process for spatial representation in young children. Nature, 370, 57-59.

Hermer, L., \& Spelke, E. (1996). Modularity and development: The case of spatial reorientation. Cognition, 61, 195-232.
Holbrook, R. I., \& Burt de Perera, T. (2011). Three-dimensional spatial cognition: Information in the vertical dimension overrides information from the horizontal. Animal Cognition, 14, 613-619. doi:10.1007/s10071-011-0393-6

Ishikawa, T., \& Montello, D. R. (2006). Spatial knowledge acquisition from direct experience in the environment: Individual differences in the development of metric knowledge and the integration of separately learned places. Cognitive Psychology, 52, 93-129.

Jacobs, L. F., \& Schenk, F. (2003). Unpacking the cognitive map: The parallel map theory of hippocampal function. Psychological Review, 110, 285-315.

Jovalekic, A., Hayman, R., Becares, N., Reid, H., Thomas, G., Wilson, J., \& Jeffery, K. (2011). Horizontal biases in rats' use of three-dimensional space. Behavioural Brain Research, 222, 279-288.

Kelly, D. M., \& Bischof, W. F. (2005). Reorienting in images of a three-dimensional environment. Journal of Experimental Psychology. Human Perception and Performance, 31, 1391-1403.

Kelly, J. W. (2011). Head for the hills: The influence of environmental slant on spatial memory organization. Psychonomic Bulletin Review, 18, 774-780. doi:10.3758/s13423-011-0100-2

Kozhevnikov, M., \& Hegarty, M. (2001). A dissociation between object-manipulation and perspective-taking spatial abilities. Memory \& Cognition, 29, 745-756.

Lavenex, P. B., \& Lavenex, P. (2010). Spatial relational learning and memory abilities do not differ between men and women in a realworld, open-field environment. Behavioural Brain Research, 207, $125-137$

Lawton, C. A. (1994). Gender differences in way-finding strategies: Relationship to spatial ability and spatial anxiety. Sex Roles, 30, 765-779.

Lawton, C. A., \& Kallai, J. (2002). Gender differences in wayfinding strategies and anxiety about wayfinding: A cross-cultural comparison. Sex Roles, 47, 389-401.

Lawton, C. A., Charleston, S. I., \& Zieles, A. S. (1996). Individualand gender-related differences in indoor wayfinding. Environment and Behavior, 28, 204-219.

Learmonth, A. E., Nadel, L., \& Newcombe, N. S. (2002). Children's use of landmarks: Implications for modularity theory. Psychological Science, 13, 337-341.

Learmonth, A. E., Newcombe, N. S., Sheridan, N., \& Jones, M. (2008). Why size counts: Children's spatial reorientation in large and small enclosures. Developmental Science, 11, 414-426.

Liben, L. S. (1995). Educational applications of geographic information systems: A developmental psychologist's perspective. In D. Barstow (Ed.), First national conference on the educational applications of geographic information systems (pp. 44-49). Cambridge, MA: TERC.

Moffat, S. D., Hampson, E., \& Hatzipantelis, M. (1998). Navigation in a "virtual" maze: Sex differences and correlation with psychometric measures of spatial ability in humans. Evolution and Human Behavior, 19, 73-87.

Nardi, D., \& Bingman, V. P. (2009). Pigeon (Columba livia) encoding of a goal location: The relative importance of shape geometry and slope information. Journal of Comparative Psychology, 123, 204-216.

Nardi, D., Newcombe, N. S., \& Shipley, T. F. (2011). The world is not flat: Can people reorient using slope? Journal of Experimental Psychology: Learning, Memory, and Cognition, 37, 354-367.

Nardi, D., Nitsch, K. P., \& Bingman, V. P. (2010). Slope-driven goal location behavior in pigeons. Journal of Experimental Psychology. Animal Behavior Processes, 36, 430-442.

Nardini, M., Jones, P., Bedford, R., \& Braddick, O. (2008). Development of cue integration in human navigation. Current Biology, 18, 689-693. 
O’Laughlin, E. M., \& Brubaker, B. S. (1998). Use of landmarks in cognitive mapping: Gender differences in self report versus performance. Personality and Individual Differences, 24, 595-601.

Piaget, J., \& Inhelder, B. (1956). The child's conception of space. London, U.K.: Routledge \& Kegan-Paul.

Ratliff, K. R., \& Newcombe, N. S. (2008). Reorienting when cues conflict: Evidence for an adaptive-combination view. Psychological Science, 19, 1301-1307.

Restat, J. D., Steck, S. D., Mochnatzki, H. F., \& Mallot, H. A. (2004). Geographical slant facilitates navigation and orientation in virtual environments. Perception, 33, 667-687.

Sandstrom, N. J., Kaufman, J., \& Huettel, S. A. (1998). Males and females use different distal cues in a virtual environment navigation task. Cognitive Brain Research, 6, 351-360.

Saucier, D. M., Green, S. M., Leason, J., MacFadden, A., Bell, S., \& Elias, L. J. (2002). Are sex differences in navigation caused by sexually dimorphic strategies or by sex differences in the ability to use strategies? Behavioral Neuroscience, 116, 403-410.

Schinazi, V. R., Epstein, R. A., Nardi, D., Newcombe, N. S., \& Shipley, T. F. (2009). The acquisition of spatial knowledge in an unfamiliar campus environment. Boston, MA: Poster presented at annual meeting of the Psychonomic Society.
Sholl, M. J. (1989). The relation between horizontality and rod-and-frame and vestibular navigation performance. Journal of Experimental Psychology: Learning, Memory, and Cognition, 15, 110-125.

Tan, D. S., Czerwinski, M. P., \& Robertson, G. G. (2006). Large displays enhance optical flow cues and narrow the gender gap in 3-D virtual navigation. Human Factors, 48, 318-333.

Vargas, J. P., Lopez, J. C., \& Thinus-Blanc, C. (2004a). Encoding of geometric and featural spatial information by goldfish (Carassius auratus). Journal of Comparative Psychology, 118, 206-216.

Vargas, J. P., Petruso, E. J., \& Bingman, V. P. (2004b). Hippocampal formation is required for geometric navigation in pigeons. European Journal of Neuroscience, 20, 1937-1944.

Voyer, D., Voyer, S., \& Bryden, M. P. (1995). Magnitude of sex differences in spatial abilities: A meta-analysis and consideration of critical variables. Psychological Bulletin, 117, 250-270.

Ward, S. L., Newcombe, N., \& Overton, W. F. (1986). Turn left at the church, or three miles north: A study of direction giving and sex differences. Environment and Behavior, 18, 192-213.

Weisberg, S. M., Nardi, D., Newcombe, N. S., \& Shipley, T. F. (2012). Sensing the slopes, Sensory modality effects in using slope for a goal location task. Manuscript under review. 